\title{
Domain Wall Dynamics in Amorphous Ferromagnetic Wire with Small Helical Anisotropy
}

\author{
J. Ziman*, M. Kladivová, J. Onufer and B. Zagyi \\ Department of Physics, Technical University of Košice, Park Komenského 2, 04200 Košice, Slovak Republic \\ Dynamics of the domain wall between circular magnetic domains in amorphous ferromagnetic \\ $\mathrm{Co}_{68.2} \mathrm{Fe}_{4.3} \mathrm{Si}_{12.5} \mathrm{~B}_{15}$ wire was studied in a small region around the domain wall equilibrium position in an \\ inhomogeneous magnetic field. The wire was prepared using the in-rotating-water-quenching technique. Small \\ helical anisotropy was induced in the wire by current annealing and simultaneous application of tensile stress and \\ torsion. The inhomogeneous magnetic field acted on domain wall by restoring force directly proportional to the \\ displacement of the domain wall from its equilibrium position. Domain wall position was monitored by circular \\ magnetic flux measurements from which it results that domain wall motion can be modeled by the motion of a \\ very strongly damped pendulum. The value of domain wall mobility obtained from these experiments is in good \\ agreement with the values calculated and measured for domain wall driven by a constant magnetic field.
}

PACS numbers: 75.60.Ch, 75.60.Jk, 75.50.Kj

\section{Introduction}

The properties of amorphous ferromagnetic wires make them suitable for the study of single domain wall (DW) dynamics. A single giant Barkhausen jump in positive magnetostrictive wires is the most frequently studied phenomenon [1-3]. In experiments presented in these works the DW separating two axial magnetic domains is driven by the axial magnetic field. The dynamics of a DW between two circular magnetic domains can be studied in wires with small or negative magnetostriction $[4,5]$. In this case the DW is driven by the circular magnetic field created by electric current flowing through the wire.

The study of the dynamics of the DW between circular magnetic domains driven by an inhomogeneous axial magnetic field in a wire with small helical anisotropy is presented in this paper.

\section{Results and discussion}

Amorphous ferromagnetic $\mathrm{Co}_{68.2} \mathrm{Fe}_{4.3} \mathrm{Si}_{12.5} \mathrm{~B}_{15}$ wire with nominal diameter of $125 \mu \mathrm{m}$, prepared using the in-rotating-water-quenching technique, was treated in two steps - furnace annealing $\left(460^{\circ} \mathrm{C}\right.$ for $3 \mathrm{~min}$ ) and current annealing ( $0.5 \mathrm{~A}$ for $3 \mathrm{~min})$ with simultaneous application of tensile stress (367 MPa) and torsion of 22 $\mathrm{rad} / \mathrm{m}$. The hysteresis loop of axial and circular magnetization versus the axial field measured after the treatment confirmed the presence of a small helical anisotropy. Deviation of the easy axis from circular direction $\gamma$ was

* corresponding author; e-mail: jan.ziman@tuke.sk

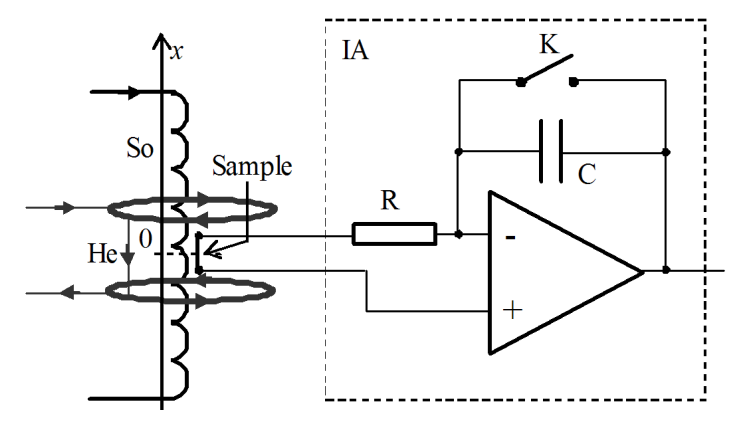

Fig. 1. Experimental setup. So - solenoid, He Helmholtz coils, IA — integrating amplifier.

estimated from the remanent axial magnetization to be $\gamma \approx 4^{\circ}$. The experimental setup used for the measurement presented in this paper is depicted in Fig. 1. Two coils (He, radius $R=7 \mathrm{~cm}$ ) in the Helmholtz geometry with opposite currents flowing through them generated an inhomogeneous magnetic field. A homogeneous magnetic field was generated by solenoid (So). Thin copper wires were attached to the ends of the sample with silver paint and then connected to the input of an integrating amplifier (IA). In this way the changes in circular magnetic flux $\Delta \Phi_{\varphi}$ could be monitored. The length $l$ of the sample was $6 \mathrm{~cm}$, so that $l<R$. The centres of He and the sample coincided. A single DW between two oppositely magnetized adjacent domains was formed when the magnitude of the inhomogeneous magnetic field was high enough (amplitude higher than $150 \mathrm{~A} / \mathrm{m}$ ). The DW was located in the middle of the Helmholtz coils $(x=0)$ for the zero homogeneous field $\left(H_{\text {So }}\right)$ generated by the 
solenoid. For positions $x$ not far from this equilibrium position (in comparison with $R$ ) the inhomogeneous field generated by the Helmholtz coils $\left(H_{\mathrm{He}}\right)$ can be considered to be directly proportional to $x\left(H_{\mathrm{He}}=-c x\right)$. The experimental setup allows an experiment in which for a given $H_{\mathrm{He}}$ the DW is displaced from its equilibrium position by the field $H_{\text {So }}$ and then after switching off the field $H_{\text {So }}$ the wall returns to its equilibrium position. This DW displacement is monitored by the measurement of $\Delta \Phi_{\varphi}$.

Theoretically (in low field approximation) this experiment can be described as follows. The net force acting on the wall is given by the formula

$$
F=m \frac{\mathrm{d}^{2} x}{\mathrm{~d} t^{2}}=2 S \mu_{0} M_{\mathrm{S}} H_{\mathrm{e}}-k_{b} v-2 S \mu_{0} M_{\mathrm{S}} H_{\mathrm{e} 0},
$$

where $S$ is area of the wall, $m$ is mass of the DW, $M_{\mathrm{S}}$ is saturation magnetization, $\mu_{0}$ is permeability of free space, $k_{b}$ is damping coefficient, $v$ is DW velocity, $H_{\mathrm{e} 0}$ is wall coercivity and $H_{\mathrm{e}}$ is an easy axis field (projection of applied axial field to the easy axis direction).

For constant homogeneous $H_{\mathrm{e}}$ the velocity satisfies the formula $v=\mu\left(H_{\mathrm{e}}-H_{\mathrm{e} 0}\right)$, where $\mu$ is the so-called wall mobility. When $H_{\mathrm{e}}$ is directly proportional to $x$ $\left(H_{\mathrm{e}} \approx H_{\mathrm{He}} \gamma=-\gamma c x=-h x\right.$, for small $\left.\gamma\right)$ the equation of motion of the DW can be obtained by rearranging Eq. (1):

$$
\frac{\mathrm{d}^{2} x}{\mathrm{~d} t^{2}}+2 b \frac{\mathrm{d} x}{\mathrm{~d} t}+\omega_{0}^{2} x=-\beta,
$$

where $b=\xi / \mu, \omega_{0}^{2}=2 \xi h, \beta=2 \xi H_{\mathrm{e} 0}$ and $\xi=\mu_{0} M_{\mathrm{S}} S / m$.

For initial conditions $x=x_{0}, v=0$ at $t=0$ and for very strong damping $\left(\omega_{0} \ll b\right)$ the solution of Eq. (2) can be expressed as

$$
x=\left(x_{0}+\frac{\beta}{\omega_{0}^{2}}\right) \mathrm{e}^{-\mu h t}-\frac{\beta}{\omega_{0}^{2}} .
$$

Since $\Delta \Phi_{\varphi} \sim x$, Eq. (3) predicts the exponential time function for $\Delta \Phi_{\varphi}(t)$, which is measured in the experiment.

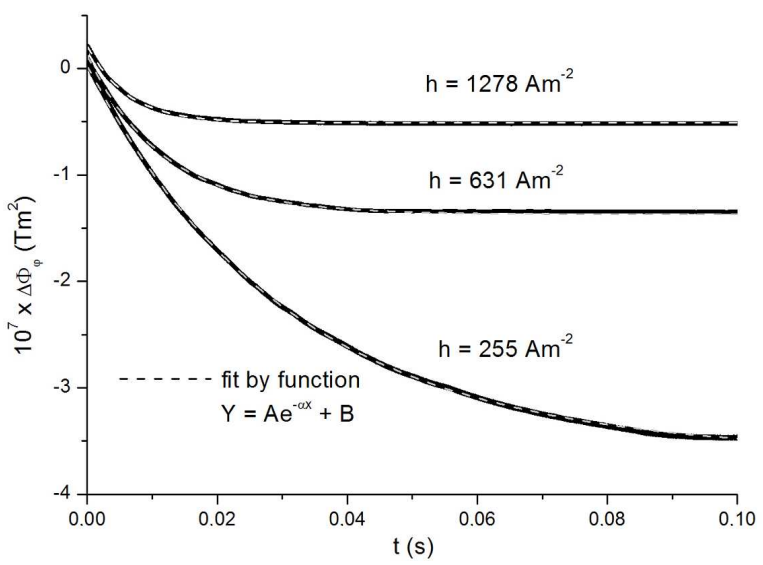

Fig. 2. Circular magnetic flux as a function of time (caused by DW returning to its equilibrium position) for three values of the slope of the inhomogeneous easy axis magnetic field $h$.

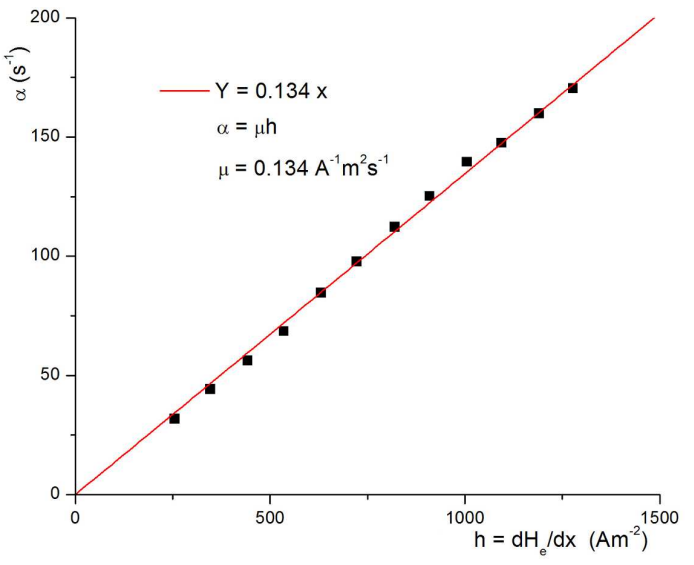

Fig. 3. Parameter $\alpha$ (see Fig. 2) as a function of the slope of the inhomogeneous easy axis magnetic field.

Typical experimental results are shown in Fig. 2. The magnitude of circular magnetization in adjacent domain slightly changes during wall motion. It can be shown (by analysis of $\Delta \Phi_{\varphi}\left(H_{\mathrm{So}}\right)$ hysteresis loop) that this contribution to measured value $\Delta \Phi_{\varphi}$ during the DW displacement is negligible (less than 1\%). Fitting the experimental curve to an exponential function (see Fig. 2 and Eq. (3)) provided the value of parameter $\alpha$. This should satisfy the relation $\alpha=\mu h$ which was experimentally confirmed (see Fig. 3). The DW mobility $\mu$ obtained in this way is in good agreement with the values calculated and measured for a DW driven by a constant magnetic field [4]. It can also be concluded that the DW motion can be modeled by the motion of a very strongly damped pendulum.

\section{Acknowledgments}

This work was supported by the Slovak Ministry of Education under projects VEGA 1/0108/09 and 1/0136/10.

\section{References}

[1] R. Varga, K.L. Garcia, M. Vázquez, P. Vojtaník, Phys. Rev. Lett. 94, 017201 (2005).

[2] R. Varga, K. Richter, A. Zhukov, V.C. Larin, IEEE Trans. Magn. 44, 3925 (2008).

[3] V. Zhukova, J.M. Blanco, M. Ipatov, R. Varga, J. Gonzales, A. Zhukov, Physica B 403, 382 (2008).

[4] J. Ziman, M. Kladivová, B. Zagyi, J. Magn. Magn. Mater. 234, 529 (2001).

[5] A.S. Antonov, N.A. Buznikov, A.B. Granovsky, A.V. Joura, A.L. Rakhmanov, A.M. Yakunin, J. Magn. Magn. Mater. 249, 95 (2002). 